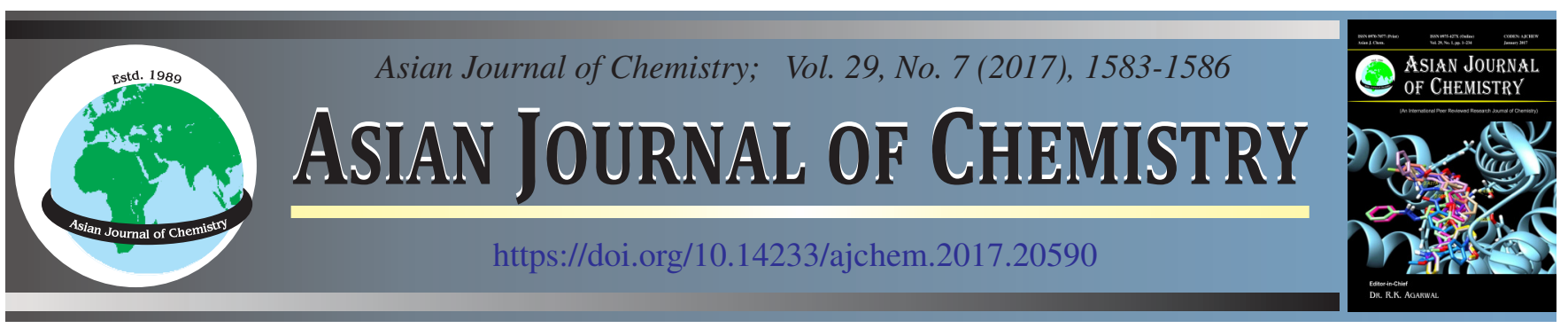

\title{
Screening of Antituberculosis Drugs by Thin Layer Chromatography
}

\author{
P. Mishra ${ }^{1}$, A. Durgbanshi ${ }^{1, *}$ and R.P. PaWAR ${ }^{2}$
}

${ }^{1}$ Department of Chemistry, Dr. Harisingh Gour Vishwavidyalaya (A Central University), Sagar-470 003, India

${ }^{2}$ Department of Criminology and Forensic Science, Dr. Harisingh Gour Vishwavidyalaya (A Central University), Sagar-470 003, India

*Corresponding author: E-mail: abhiasha126@gmail.com

Tuberculosis remains a major public health problem in developing countries. This work demonstrated a new, cost effective, fast and reproducible thin layer chromatographic screening method for the determination of rifampicin, isoniazid and pyrazinamide in pharmaceutical dosages. The solvent system used for the determination of these three drugs is (propanol:hexane:ethyl acetate:ammonia) (5:2:1.7:0.2 v/v) and the $R_{f}$ value of rifampicin, pyrazinamide and isoniazid was $0.68,0.74$ and 0.50 , respectively. The intra-day and inter-day repeatability of the developed method for retention factor showed standard deviation (SD) of approximately 0.5-1.0\%.

Keywords: Rifampicin, Isoniazid, Pyrazinamide, Thin layer chromatography.

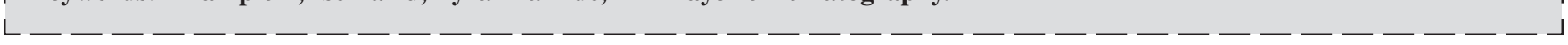

\section{INTRODUCTION}

Rifampicin (RIF), pyrazinamide (PYZ) and isoniazid (INH) are categorized as first line antituberclular agent. These drugs are widely available in the market for the medication of tuberculosis which still is a serious communicable disease in developing and under develop countries caused commonly by Mycobacterium tuberculosis [1]. During the treatment these three drugs are given in combination and in due of time course rifampicin is continued while pyrazinamide and isoniazid are either discontinued or given alternately.

Rifampicin is chemically known as 4-methyl-1-perazinaminyl (Table-1). It is a semisynthetic antibiotic derived from Rifamycin B which is obtained from Streptomyces mediterranei used for the treatment of tuberculosis and other infectious diseases [2].

The action of rifampicin may be attributed to inhibit bacterial RNA by binding to the $\beta$ subunit of DNA dependent RNA polymerase, thus blocking RNA transcription [3]. It is freely soluble in chloroform, methanol and slightly soluble in water [4,5].

Isoniazid is another common antibiotic prescribed for the treatment of tuberculosis and it is chemically known as pyridine-4-carbohydrazide (Table-1). It is generally used in combination with other antibiotic, because of its tendency to develop antibacterial resistance. The antibacterial activity of isoniazid is due to the fact that it inhibits the synthesis of mycolic acid which is an essential component of mycobacterial cell walls. It is freely soluble in water, methanol and also in chloroform [6].
Together with rifampicin and isoniazid, pyrazinamide is also used to treat active tuberculosis. It is chemically known as pyrazine-2-carboxamide (Table-1). Its mode of action is completely different from rifampicin and isoniazid as it kills the semi-dormant Tubercle bacilli in acidic media which is not possible by using any other antituberculosis drug [7].

The three drugs are administrated together in a combination generally rifampicin $(450 \mathrm{mg})$, isoniazid $(300 \mathrm{mg})$ and pyrazinamide $(750 \mathrm{mg}$ ) [5]. The dosage is available in tablet or capsule.

As tuberculosis is very common and prevalent diseases and most of the treatment are through government agencies and hospitals. The government tuberculosis hospitals provide these medicines free of cost so they are produced and stored in bulk. Therefore there is an urgent need to check the stability and the contents of these formulations from time to time before being distributed among patients. There are various analytical techniques which can be used for the separation and detection of these drugs in formulations. Various quantitative and qualitative methodologies based on spectroscopy (UV visible spectrophotmetry for the determination of rifampicin and pyrazinamide in pharmaceutical formulation) $[8,9]$. Similarly, high performance liquid chromatography (HPLC) can be used to separate these drugs in pharmaceutical formulation as well as for therapeutic monitoring of these drug in tubercular patient [10,11], high end sophisticated instrumentation like LC-MS or LC-MS/MS have been recently used to study the metabolic ratio of these drugs in biological fluids or for quantification of these drugs in CSF $[12,13]$. High performance thin layer 
TABLE-1

STRUCTURES pKa AND log P VALUE OF THE THREE DRUGS STUDIED

Compound

chromatography (HPTLC) is also a separation technique which can also be used for the determination and quantification of these drugs in pharmaceutical formulation [6]. All the above mentioned technique was reported for the determination and quantification of these drugs in pharmaceutical formulation as well as in biological fluid. However, the modern techniques are fast, reliable but to some extent they are expensive also. On one hand spectroscopic technique is low-cost easy to use and sensitive but it lacks selectivity where as high end instrumentation could not be used for routine analysis or for the purpose of screening as they need trained person for the handling of these sophisticated instruments.

Among all the above analytical techniques thin layer chromatography (TLC) is a simple, cost effective, fast and reliable technique for the screening of the pharmaceutical drugs. Effort has been made using thin layer chromatographic technique for the separation and identification of rifampicin, isoniazid and pyrazinamide in the fixed dose combinations. The present research work deals with the development of simple thin layer chromatographic technique for the determination of rifampicin, isoniazid and pyrazinamide.

\section{EXPERIMENTAL}

Pre-coated silica gel $60 \mathrm{~F}_{254}, 20 \times 20 \mathrm{~cm}$ TLC plate was purchased from E. Merck (India) Limited, (Mumbai, India). The plates were developed in horizontal twin trough glass chamber. Standard samples of rifampicin, pyrazinamide and isoniazid were gifted by Wilcure Remedies Pvt. Limited, (Indore, India). The other pharmaceuticals product P-Zide 500 Cadila Pharmaceutical Limited, (Gujrat, India), PZA-CIBA,
Sandoz Pvt. Ltd (Novartis India), Z = pyrazinamide (Lupin Limited) Pyrazinamide Forecox (Macleods Pharmaceutical Ltd). Analytical grade chemicals i.e. acetone, cyclo-hexane, toluene, carbon tetra chloride, glacial acetic acid, hydrochloric acid were purchased from Qualigens Fine Chemicals, (Mumbai, India), methanol, ethanol, propanol, butanol, chloroform, ammonia were purchased from Merck, (Mumbai, India). Double distilled water was prepared using a borosil distillation unit (Borosil Glass Works Limited, (Mumbai, India). The standards were weighted using an analytical balance Mettler Toledo (Mettler Toledo India Pvt. Ltd. India). To dissolve the samples Ultrasonic bath (Sonicator) 1.5 lt (PCI Analytics Pvt. Ltd. Russia) and Deepfreezer (Remi electrotechnik Limited India) were used. All the solution were filtered through 0.45 $\mu \mathrm{m}$ nylon membrane (Micron Separation, Westboro, MA, USA). The sample was spotted on TLC plate using 1-10 $\mu \mathrm{L}$ micropipette purchased from Tarson (Kolkata, India). A UV cabinet model 'CRYSTAL 3' (Mumbai, India) visualizer containing 254 and $365 \mathrm{~nm}$ range was used for visualization.

Sample preparation: $1000 \mathrm{ppm}$ stock solution of reference standards of rifampicin, pyrazinamide and isoniazid was prepared by dissolving in methanol and water stored in amber glass volumetric flask under frozen condition.

For the analysis of tablets, each tablet was weighed accurately and then crushed using a pestle-mortar. The powder was again weighed and finally $10 \mathrm{mg}$ of each powered tablet was dissolved in different solvents. Rifampicin was dissolved in $10 \mathrm{~mL}$ of methanol whereas pyrazinamide and isoniazid were dissolved in water and ultrasonicated for $10 \mathrm{~min}$. After dissolution the entire sample was filtered through Whatman filter paper and stored for later use. 
TLC procedure: $1 \mu \mathrm{L}$ standard solutions of the pyrazinamide, isoniazid and rifampicin along with the real samples (pharmaceutical preparations) were spotted using a micropipette on a TLC plate. The distance of the spots from the edges of the plate was $1.5 \mathrm{~cm}$ and the distance between two spot was $1 \mathrm{~cm}$. Many solvent systems were taken in to consideration based on the elutropic series of solvents and the property of the analyte.

According to the requirement clean glass chromatographic chambers were taken for the development of the TLC plate. The chamber containing different solvent system was saturated for $30 \mathrm{~min}$. Solvent front was marked $10 \mathrm{~cm}$ from the spotting point and the loaded plate was developed in ascending way. When the mobile phase had travelled the required distance, the plates were taken out from the chamber and the solvent front was marked. Then the plates were air dried for $15 \mathrm{~min}$ at room temperature.

After proper drying the plates were viewed under ultra violet light in a visualization chamber at 254 and $365 \mathrm{~nm}$ wavelength and the photographs of each developed TLC plates were taken using a digital camera.

\section{RESULTS AND DISCUSSION}

Optimization of mobile phase: The first step for getting a good resolution and proper selectivity is the optimization of separation conditions which may include various parameters. One of the parameters being optimization of the mobile phase which gives best result for the separation of rifampicin, pyrazinamide and isoniazid. Different solvents according to elutropic series in different ratio were tried for getting best separation in minimum time.

Firstly, in non-polar solvent hexane was chosen because hexane is environmentally less hazardous as compared to the other non-polar solvent like benzene, acetonitrile, toluene, carbon tetrachloride etc. In $100 \%$ hexane spot of pyrazinamide and isoniazid did not move whereas rifampicin moved very fast. In $100 \%$ propanol which is slightly polar in nature spot of rifampicin is retained on stationary phase and both pyrazinamide and isoniazid moved with solvent front. So, it is established that rifampicin is non-polar and isoniazid and pyrazinamide are relatively polar compounds (Table-1). In methanol and water the mobility of pyrazinamide and isoniazid were very fast as they travelled with the solvent front and the mobility of rifampicin is very poor. Likewise when pure ethyl acetate, a moderately polar solvent was used rifampicin was strongly retained on the plate and isoniazid and pyrazinamide very slowly. After trying all the above mentioned solvents finally the solvent system chosen was propanol: hexane: ethyl acetate: ammonia. Different ratios of the solvent system were tried and the solvent system that gave best separation of the three drugs was (propanol:hexane:ethyl acetate:ammonia) $(5: 2: 1.7: 0.2)$.

In order to increase the spot efficiency and to reduce the tailing effect a small amount of ammonia is used in the solvent system. The reason for the increase in spot efficiency and decrease in tailing effect is the decreased viscosity of ammonia which when added to the solvent system decreases the overall viscosity of the solvent system thereby increasing the rate of movement of the mobile phase through the stationary phase and hence reducing the diffusion of the drugs on to the stationary phase. When the optimum mobile phase was used the $\mathrm{R}_{\mathrm{f}}$ value of rifampicin, pyrazinamide and isoniazid obtained was $0.68,0.74$ and 0.50 , respectively (Fig. 1).

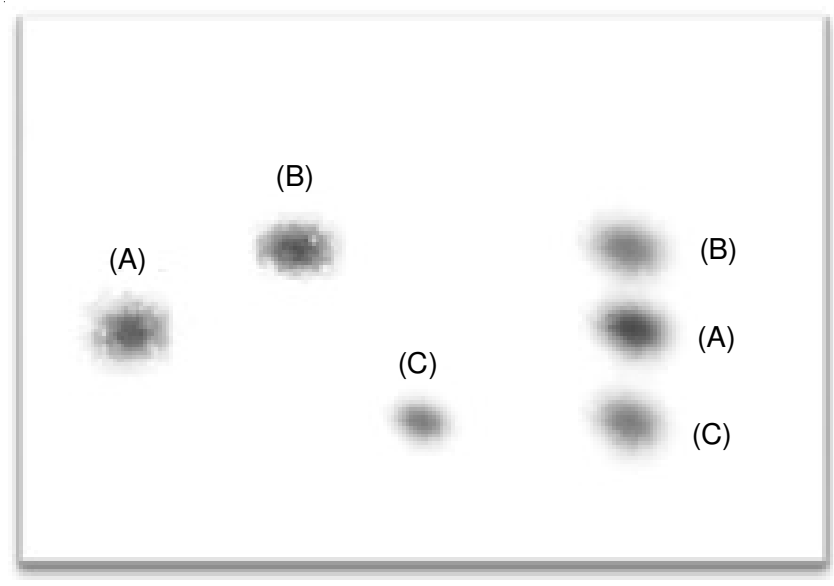

Fig. 1. Thin layer chromatogram of standard drugs - (A) rifampicin, (B) pyrazinamide and (C) isoniazid, developing solvent (propanol: hexane:ethyl acetate:ammonia) (5:2:1.7:0.2)

Limit of detection, repeatability and reproducibility: The sensitivity of any analytical method is the most important parameter and the term which defines it is limit of detection (LOD). In order to calculate the limit of detection different concentrations of all the three drug were prepared from 1 to $500 \mu \mathrm{g} / \mathrm{mL}$. The minimum concentration which could be visualized under UV visualization chamber was 10, 85 and $111 \mu \mathrm{g} /$ $\mathrm{mL}$ for rifampicin, pyrazinamide and isoniazid, respectively.

For repeatability and reproducibility study, the spots of three different concentrations i.e. 50, 100 and $150 \mu \mathrm{g} / \mathrm{mL}$ rifampicin, pyrazinamide and isoniazid of the three drugs run in the optimum mobile phase. In order to carry out inter-day repeatability 5 TLC separation of the three drugs were carried out on seven different days and the difference in retention factors were studied (Table-2). Similar TLC separations were performed for intra-day repeatability and 5 run of the three drugs were made on the same day. The difference in retention factor was noted down (Table-3). The experiments were carried out on intra- and inter-day in inter laboratory basis spread over a period of two months. All the experimental results was almost similar, hence it could be concluded that the developed method was stable.

\begin{tabular}{cccc}
\multicolumn{4}{c}{ TABLE-2 } \\
\multicolumn{4}{c}{ INTER-DAY REPEATABILITY } \\
\hline Day & Rifampicin $\left(\mathrm{R}_{\mathrm{f}}\right)$ & Pyrazinamide $\left(\mathrm{R}_{\mathrm{f}}\right)$ & Isoniazid $\left(\mathrm{R}_{\mathrm{f}}\right)$ \\
\hline $1^{\text {st }}$ & 0.68 & 0.74 & 0.50 \\
$2^{\text {nd }}$ & 0.80 & 0.74 & 0.48 \\
$3^{\text {rd }}$ & 0.80 & 0.80 & 0.51 \\
$4^{\text {th }}$ & 0.90 & 0.89 & 0.64 \\
$5^{\text {th }}$ & 0.99 & 0.72 & 0.71 \\
$6^{\text {th }}$ & 0.99 & 0.85 & 0.71 \\
$7^{\text {th }}$ & 0.85 & 0.81 & 0.58 \\
SD & 0.11 & 0.06 & 0.09 \\
\hline
\end{tabular}




\begin{tabular}{cccc}
\hline \multicolumn{4}{c}{ TABLE-3 } \\
\multicolumn{4}{c}{ INTRA-DAY REPEATABILITY } \\
\hline TLC & Rifampicin $\left(\mathrm{R}_{\mathrm{f}}\right)$ & Pyrazinamide $\left(\mathrm{R}_{\mathrm{f}}\right)$ & Isoniazid $\left(\mathrm{R}_{\mathrm{f}}\right)$ \\
\hline $1^{\text {st }}$ & 0.68 & 0.74 & 0.50 \\
$2^{\text {nd }}$ & 0.68 & 0.80 & 0.61 \\
$3^{\text {rd }}$ & 0.71 & 0.89 & 0.72 \\
$4^{\text {th }}$ & 0.80 & 0.90 & 0.81 \\
$5^{\text {th }}$ & 0.88 & 0.90 & 0.81 \\
$\mathrm{SD}$ & 0.08 & 0.07 & 0.13 \\
\hline
\end{tabular}

\section{Conclusion}

A rapid, sensitive, cost effective, ready to use, screening method was developed for the simultaneous determination of most frequently used antituberculosis drugs using thin layer chromatographic separation technique. The optimum mobile phase was found to be propanol:hexane:ethyl acetate:ammonia $(5: 2: 1.7: 0.2 \mathrm{v} / \mathrm{v})$ and the $\mathrm{R}_{\mathrm{f}}$ value of rifampicin, pyrazinamide and isoniazid was $0.68,0.74$ and 0.50 , respectively. The developed method was successfully applied to pharmaceutical sample containing rifampicin, pyrazinamide and isoniazid having good repeatability and reproducibility. Limit of quantification was found out to be $10 \mathrm{ppm}$ for it. The developed method could be used as a screening technique of rifampicin, pyrazinamide and isoniazid and separation before proceeding this compound for sophisticated instrumental technique for confirmation.

\section{ACKNOWLEDGEMENTS}

The authors are thankful to Madhya Pradesh Council \& Technology (MPCOST), Bhopal, Madhya Pradesh (India) and University Grants Commission (UGC), New Delhi (India) for providing Junior Research Fellowship.

\section{REFERENCES}

1. K. Ilango and S. Arunkumar, Rasayan J. Chem., 3, 493 (2010).

2. K. Florey, Analytical Profiles of Drug Substances, Academic Press, New York, p 467 (2005).

3. S. Somasundaram, A. Ram and L. Sankaranarayanan, J. Tuber. Res., 2, 40 (2014); https://doi.org/10.4236/jtr.2014.21005.

4. Indian Pharmacopoeia, The Controller Publication, Government of India, New Delhi, vol. 3, p. 2054 (2010).

5. K.D. Tripathi, Essentials of Medical Pharmacology, Japee Brothers Medical Publishers (P) Ltd., New Delhi, vol. 6, p. 740 (2008).

6. S. Puthusseri and M. Mathew, World J. Pharm. Res., 3, 523 (2014).

7. Y. Zhang, A. Scorpio, H. Nikaido and Z. Sun, J. Bacteriol., 181, 2044 (1999).

8. B.R. Shankar, R. Rajesh and K. Ramya, Asian J. Pharm. Anal. Med. Chem., 8, 4 (2016).

9. G.P. Chenna, S.K. Shetty, J.B. Pai, B. Gopinath and M. Ahmed, Int. J. Chemtech Res., 3, 737 (2011).

10. H. Wang, C. Cai, C. Chu, J. Liu, Y. Kong, M. Zhu and T. Zhang, Asian J. Pharm. Sci., 7, 303 (2012).

11. S. Unsalan, M. Sancar, B. Bekce, P.M. Clark, T. Karagoz, F.V. Izzettin and S. Rollas, Chromatographia, 61, 595 (2005); https://doi.org/10.1365/s10337-005-0549-0.

12. S.H. Song, S.H. Jun, K.U. Park, Y. Yoon, J.H. Lee, J.Q. Kim and J. Song, Rapid Commun. Mass Spectrosc., 21, 1331 (2007); https://doi.org/10.1002/rcm.2961.

13. A. Srivastava, D. Waterhouse, A. Ardrey and S.A. Ward, J. Pharm. Biomed. Anal., 70, 523 (2012);

https://doi.org/10.1016/j.jpba.2012.05.028. 